\title{
La educación ambiental como una filosofía de vida
}

\section{Environmental Education as a Life Philosophy}

\author{
Julio Coutiño Molina ${ }^{I}$ \\ Tuxtla Gutiérrez \\ Chiapas, México \\ jcoutino@ecosur.mx
}

Recibido 08 de junio de 2011 • Aceptado 26 de agosto de 2011

\begin{abstract}
Resumen. La educación ambiental (E. A.) resulta de gran importancia en la conservación del medio ambiente al transmitir valores acordes al desarrollo sustentable. Sin embargo, ante el actual paradigma ambiental cabe preguntar: ¿es necesario abordar la E. A. desde una perspectiva más amplia o nuestra visión de ella es reduccionista? La relación de las personas con el medio ambiente necesita adecuarse a la problemática. Esto implica que los esfuerzos y principios de la E. A. deberían adoptarse a nuestra vida cotidiana y aplicarse en esta, haciendo de ella una filosofía de vida, derivada del fuero interno de cada persona, meditada y basada en principios éticos. De esa manera podrían lograrse acciones y actitudes más responsables y comprometidas, que nos beneficien con su cuidado. Un aporte quizá pequeño pero continuo.
\end{abstract}

Palabras claves. Ambiente, cultura, ecología, ética, responsabilidad, sociedad.

Abstract. Environmental education (E. E.) is of great importance in preserving the environment, transmitting values based on sustainable development. However, given the current environmental paradigm, we must reflect on the question: is it necessary to approach E. E. from a broader perspective or are we seeing E. E. from a narrow perspective? People's relationship with the environment needs to change. This means that the efforts and principles of E. E. should be adopted and applied in our daily live, making it a philosophy of life, deep inside each person, thoughtful and based on ethical principles. Thus more responsible and committed actions and attitudes could be achieved, which would contribute to environmental care. This may be a small, but continuous contribution.

Keywords. Culture, Ecology, environment, ethics, responsibility, society.

\footnotetext{
Maestro en Ciencias en Recursos Naturales y Desarrollo Rural por El Colegio de La Frontera Sur. Licenciado en Biología egresado de la Universidad de Ciencias y Artes de Chiapas. He laborado como educador ambiental en el Parque Ecoturístico Cañón del Sumidero.
} 


\section{Introducción}

La educación ambiental como un esfuerzo encaminado a la conservación del medio ambiente resulta de gran valía al transmitir, a los individuos y sociedades, los valores acordes con el desarrollo sustentable. La educación ambiental (en adelante E. A.) se definió, en diversos foros y congresos (v. gr. Moscú, en 1987, y el Foro Global Ciudadano de 1992, realizado en Río de Janeiro), como un proceso de aprendizaje en el que individuos y comunidades adquieren conciencia de su entorno, la cual les permite actuar en la resolución de las problemáticas inherentes presentes y futuras (Guillén, 1996; Novo, 1996). Incluye, dentro de su cuerpo de conocimientos, una serie de acciones tales como la separación de desechos y el reciclaje, la reducción en el consumo de productos no renovables, el ahorro en el consumo energético, entre otras. Sin embargo, es necesario considerar si este conjunto de esfuerzos, por sí solos, son suficientes para hacer frente al actual paradigma ambiental, es decir: ¿es necesario abordar la E. A. desde una perspectiva más amplia que la de un solo conjunto de definiciones y métodos?

\section{La educación ambiental como una filosofía de vida}

Un sujeto camina por la calle y deja en la acera el envase del refresco que ha terminado de beber. Mientras tanto, frente a él, tras de un cristal alguien más come una hamburguesa. En otro lado, otra persona sale de su hogar y deja su computadora conectada a la toma de corriente y el grifo del agua goteando. ¿Acaso se ignora que las inundaciones en las ciudades son, en gran parte, debido a la acumulación de PET (polietileno tereftalato), uno de los materiales plásticos más comunes en la elaboración de botellas y empaques) en los sistemas de drenaje y alcantarillado? ¿O que la demanda de carne de las grandes trasnacionales de comida rápida genera la conversión de grandes extensiones de tierra con fines de pastoreo y que parte de sus insumos provienen de cultivos transgénicos? ¿Quizá no saben que la construcción de hidroeléctricas altera totalmente los ecosistemas? La respuesta parece ser obviamente no y, sin embargo, la mayoría de las personas tiene un concepto acertado sobre las acciones que son correctas (o mejor dicho, responsables), en cuanto al medio ambiente. Entonces, ¿han fallado los esfuerzos de la E. A o la visión que se tiene de la misma es reduccionista?

De acuerdo con Herskovits (1981), la relación entre el ser humano y su hábitat varía en la medida en que responda a las exigencias que el medio físico le imponga. Esto significa que, actualmente, el ser humano necesita responder no solo a las exigencias, sino también a la realidad del medio. No obstante, considerando que de esta relación se derivan "modos de vida" y nuevas "necesidades" (Marx y Engels, 1980), dichos cambios se perciben lejanos, si se toman en cuenta los actuales patrones de consumo, la demanda de recursos y las pautas culturales de la sociedad, que conducen a los individuos hacia actitudes inadecuadas con la conservación del ambiente.

Esto implica que los esfuerzos de la E. A. tienen que considerarse desde una perspectiva humana más amplia, con esfuerzos constantes en la cotidianidad, es decir, hacer de la E. A. una filosofía de vida, no en un sentido metafísico, sino el de una idea fuerza. Una filosofía entendida como la aplicación diaria de los principios de conservación y aprovechamiento sustentable de los recursos naturales, derivada del fuero interno de cada individuo, transmitida a manera de la técnica 
de bola de nieve (Sierra-Bravo, 1994) en todos los ámbitos de socialización de la vida diaria, y no exclusivamente en el académico (Arias, González y Benayas del Álamo, 2008).

Desde la perspectiva anterior, si la sociedad genera sus modos de vida, también puede modificarlos y renunciar al estatus de prestigio que puede significar (Engels, 1980; Marx y Engels, 1980). Es decir, la familia Pérez bien puede ir a vacacionar en una cooperativa que impulsa el desarrollo sustentable a través del ecoturismo, en vez de ir a un desarrollo hotelero que arrasa con los manglares. El quid es tratar de modificar estos patrones comenzando en cada individuo, no por obligación ni mecánicamente como cualquier otra de las actividades diarias (Bendala y Pérez, 2004), sino por un acto de responsabilidad meditada y basada en principios éticos. Se obtendría, así, bienestar mediante el cuidado del medio (Kellert y Wilson, 1993).

\section{La educación ambiental como un proceso ético}

Según Novo (1996), la E. A. es, antes que nada, un movimiento ético que no se centra exclusivamente en el aspecto conservacionista. La ética plantea un carácter eminentemente racional y normativo que orienta y dirige las decisiones libres del ser humano en su búsqueda de lo correcto (Gutiérrez, 1992). Esta capacidad de meditar en las propias acciones se manifiesta sobre todo al enfrentarse a situaciones nuevas (Linton, 1972), lo cual implica que, en vista del panorama ambiental actual, el se necesita actuar y comenzar a repetir los patrones responsables hacia el ambiente y transmitirlos a las nuevas generaciones. Ergo, ante la "nueva" situación ambiental, es necesario que la especie humana "aprenda" a actuar en consecuencia.

Lo anterior significa que la E. A. debiera transmitirse con un fuerte componente ético y no solo como un conjunto de metodologías aisladas entre sí. Esto normaría nuestras acciones sobre el ambiente, y permitiría elegir libre y responsablemente aquellas compatibles con el entorno. No se trata de decidir por otros en cuanto a sus actos para con el ambiente, sino decidir cada quien, con base en un análisis del estado de su entorno y su problemática, la manera de coadyuvar en su solución (Meza-Aguilar, 1992). Es decir, cualquiera puede participar en una marcha que contribuye a mayores emisiones vehiculares, si eso forma parte de su papel social (Linton, 1972), pero aún estas actitudes pueden modificarse como resultado de cambios de conducta más responsables, en aras de un interés mayor y de la importancia de este en la mejora del ambiente. No obstante, no se busca que la E. A. propuesta aquí como una filosofía de vida se base en principios espirituales o visiones ambientales románticas -ética biocentrista- (Chávez, 2004), sino que sea producto de la reflexión y el compromiso (compromiso denominado ética de vida por Elliot, 1995).

Hasta este punto solo se han mencionado las acciones éticas que individualmente se pueden adoptar y que dependen de la propia resolución; se han dejado de lado aquellas ajenas a la intervención inmediata. Por ejemplo, se puede pronunciar y actuar a favor o en contra de las políticas ambientales; pero la toma inmediata de decisiones consideradas éticamente correctas, se escapan de la decisión propia. Sin embargo, precisamente adoptando los principios de la E. A. como filosofía de vida, se pueden generar cambios que, desde lo individual y local, puedan transcender a lo colectivo y global (Chávez, 2004; Sánchez, 2006).

En conclusión, se puede considerar que la E. A. necesita abordarse desde un contexto de mayor responsabilidad y compromiso personal, derivado de una seria reflexión y no solo como un 
cúmulo de enseñanzas o ecotecnias. Ello influirá en las acciones de cada individuo hacia el medio ambiente, las cuales, a su vez, tendrán impacto sobre su sociedad. En caso contrario, si no se vincula de un modo más profundo la relación de las personas con el cuidado de su entorno, de nada servirá tomar "conciencia ecológica". Es necesario adoptar las medidas de la E. A. a un nivel de filosofía de vida que recuerde la responsabilidad que cada quien debe mostrar y ejercer. Un problema global como el ambiental requiere de la colaboración cotidiana, quizá pequeña, pero continua.

\section{Referencias bibliográficas}

Arias, M. A., González, E. y Benayas del Álamo, J. (2008). Educación ambiental y sociedad civil en México: Un primer apunte sobre sus prácticas pedagógicas. En F. Reyes y M. T. Bravo (Coords.). Educación ambiental para la sustentabilidad en México. Aproximaciones conceptuales, metodológicas y prácticas (pp. 187-204). Chiapas: Universidad de Ciencias y Artes de Chiapas. Recuperado de anea.org.mx/docs/EdAmbSustentabilidadMexico.pdf

Bendala, M. y Pérez, J. A. (2004). Educación ambiental: Praxis científica y vida cotidiana. Descripción de un proyecto. Revista Eureka sobre enseñanza y divulgación de las ciencias, 1(3), 233-239.

Chávez, M. T. (2004). La ética ambiental como reflexión en el marco de la educación en ciencias y en tecnología: hacía el desarrollo de la conciencia de la responsabilidad. Educere. La Revista Venezolana de Educación, 8(27), 483-488. Recuprado de http://www.redalyc.org/src/inicio/ ArtPdfRed.jsp?iCve $=35602706$

Elliot, R. (1995). La ética ambiental (Capítulo 24). En P. Singer (Ed.). Compendio de ética (pp. 391-404). Madrid: Alianza.

Engels, F. (1980). El origen de la familia, la propiedad privada y el estado (Obras escogidas). Moscú: Progreso.

Guillén, F. C. (1996). Educación, medio ambiente y desarrollo sostenible. Revista Iberoamericana de Educación, 11, 103-110.

Gutiérrez, R. (1992). Introducción a la ética. México: Esfinge.

Herskovits M. (1981). El hombre y sus obras. México: Fondo de Cultura Económica.

Kellert, S. y Wilson, E. (1993). The Biophilia Hypothesis [La Hipótesis de la Biofilia]. Washington, DC: Island Press.

Linton, R. (1972). Estudio del hombre (3ª ed). México: Fondo de Cultura Económica. 
Marx, K. y Engels, F. (1980). Oposición entre las concepciones materialistas e idealistas (Cap. 1). En C. Marx y F. Engels (Aut.). Obras escogidas (Tomo 1, pp. 4-39). Moscú: Progreso. Recuperado de http://es.scribd.com/doc/22921080/Marx-K-y-Engels-F-Obrasescogidas-vol-1-ed-Progreso-1980

Meza-Aguilar, L. (1992, noviembre-diciembre). Educación ambiental. ¿Para qué? Nueva Sociedad, $122,176-185$.

Novo, M. (1996). La educación ambiental formal y no formal: Dos sistemas complementarios. Revista Iberoamericana de Educación, 11, 75-102.

Sánchez, J. (2006, mayo). Los retos de lo local en lo global: Aportes analíticos y normativos. Iconos. Revista de Ciencias Sociales, 25, 77-88. Recuperado de http://redalyc.uaemex.mx/src/inicio/ ArtPdfRed.jsp?iCve $=50902508$

Sierra-Bravo, R. (1994). Técnicas de investigación social ( $8^{\mathrm{a}}$ ed). Madrid: Paraninfo. 